\title{
3 Research Suare \\ A Case of Two Shunts In The Endovascular Treatment of Type II Abernethy Syndrome
}

\section{Adam Alli \\ University of Kansas Medical Center}

Brenden Bombardier ( $\nabla$ bbombardier@kumc.edu )

University of Kansas Medical Center https://orcid.org/0000-0003-0830-2903

\section{Aaron Rohr}

Kansas Medical Center

\section{Zachary Collins}

Kansas Medical Center

\section{Kavi Raval}

Kansas Medical Center

\section{Research Article}

Keywords: Abernethy Syndrome, portosystemic shunt, portal vein, shunt embolization, venous malformations

Posted Date: September 7th, 2021

DOl: https://doi.org/10.21203/rs.3.rs-809899/v1

License: (c) (i) This work is licensed under a Creative Commons Attribution 4.0 International License. Read Full License

Version of Record: A version of this preprint was published at CVIR Endovascular on January 5th, 2022. See the published version at https://doi.org/10.1186/s42155-021-00279-7. 


\section{Abstract}

\section{Background:}

Abernethy malformation is a rare condition defined by a congenital extrahepatic portosystemic shunt, often leading to absence or hypoplasia of the intrahepatic portal venous system. Although there are no consensus treatment guidelines, interventional techniques now offer minimally invasive treatment options for Abernethy malformations. This case report describes a case of Abernethy Syndrome Type II where the patient had two separate extrahepatic portosystemic shunts treated with endovascular occlusion with two Amplatzer plugs and demonstrates the feasibility of this treatment for this rare condition. This case was in a young adult, adding to the scarce literature of treatment for Abernethy syndrome in the adult population.

\section{Case Presentation:}

We report a case of a 20-year-old female patient with neurocognitive behavioral difficulty, voracious appetite, and chronic encephalopathy secondary to type II Abernethy malformation with not one, but two extrahepatic portosystemic shunts. The patient had failed medical management and was not a liver transplant candidate. Therefore, she presented to us for an endovascular treatment option. The two shunts were treated with endovascular occlusion using Amplatzer vascular plugs. Following embolization, flow into the hypoplastic portal vein improved with near complete occlusion of flow into the portosystemic shunts, thus restoring blood flow into the native portal system. At 3 month follow up, a CT demonstrated complete occlusion of the two portosystemic shunts, and a portal vein diminutive in caliber. The portal vein measured $7 \mathrm{~mm}$ in diameter on both pre and post-procedure CT scans. The total volume of the liver was found to be $843 \mathrm{~cm} 3$ on pre-procedure CT \& $1191 \mathrm{~cm} 3$ on post-procedure CT.

\section{Conclusions:}

This report demonstrates the feasibility of using endovascular embolization to treat Abernethy II malformations. The management strategy of Type II Abernethy Syndrome should be to redirect blood flow into the hypoplastic native portal system, allowing for physiologic hepatic metabolism of splanchnic blood, hypertrophy of the portal system, and growth of the liver from the increased trophic flow.

\section{Background}

Abernethy malformation is a rare condition defined by a congenital extrahepatic portosystemic shunt, often leading to absence or hypoplasia of the intrahepatic portal venous system. Type I malformations are characterized by a congenital absence of the portal vein and end to side complete diversion of portal blood into the systemic circulation. Type II malformations are characterized by a hypoplastic portal vein and side to side partial diversion of portal blood into the systemic circulation.[1-2] Although there are no consensus treatment guidelines, Abernethy malformations are now most commonly treated by endovascular methods. This report describes a case of a type II Abernethy malformation with not one, but 
two extrahepatic portosystemic shunts, treated with endovascular occlusion. This case report was exempt from institutional board approval.

\section{Case Presentation}

A 20-year-old female patient presented with neurocognitive behavioral difficulty, voracious appetite, and chronic encephalopathy. Despite maximal medical treatment, the patient had persistently elevated serum ammonia levels, with a pre-operative level of $98 \mathrm{mcmol} / \mathrm{L}$, along with clinical signs and symptoms of encephalopathy. Contrast-enhanced computed tomography (CT) demonstrated a hypoplastic intrahepatic portal venous system. The superior mesenteric vein and splenic vein joined to form a short extrahepatic portal vein, with dominant flow into two parallel shunts into the suprahepatic inferior vena cava (IVC) (Fig. 1a). The patient was previously evaluated for candidacy for a liver transplant at a local children's hospital, but was denied eligibility. Thus, the patient and her family agreed to an endovascular procedure to increase blood flow into the intrahepatic portal venous system and decrease blood flow through the portosystemic shunts.

The embolization of the patient's two extrahepatic portosystemic shunts was performed via a right internal jugular approach. The IVC was selected with an 0.035" Amplatz wire (Boston Scientific, Marlborough, Massachusetts) with the aid of a 5F JB1 catheter (Angiodynamics, Latham, New York). The catheter was then removed, and a 10F RAABE Flexor sheath (Cook Medical, Bloomington, Indiana) was advanced into the IVC. An inferior vena cavagram was performed, demonstrating wide patency. Next, a JB1 catheter and a .035 angled glidewire (Terumo, Tokyo, Japan) were used to select the right hepatic vein. Digital subtraction angiography (DSA) was performed demonstrating a patent right hepatic vein. The left hepatic vein was then selected, demonstrating a massively dilated vein. Next the portal vein was selected and venography was performed, confirming dominant flow into shunts and a small caliber portal vein. The splenic vein, inferior mesenteric vein, and superior mesenteric vein were all evaluated and were patent with flow into the two portosystemic shunts rather than the diminutive portal vein (Fig. 1b). Next, an occlusion balloon (Edwards LifeSciences, Irvine, California) was inflated in the larger shunt to measure pressures prior to plug deployment. The IVC pressure measured $16 \mathrm{mmHg}$, while the portal venous pressure with occlusion of the shunt measured $18 \mathrm{mmHg}$ prior to intervention. A $16 \mathrm{~mm}$ AVPII Amplatzer plug (Abbot, Chicago, Illinois) was deployed in the larger portosystemic shunt. After selection of the second shunt, an occlusion balloon was again inflated, and the portal venous pressure was found to be $18 \mathrm{mmHg}$. Next a $12 \mathrm{~mm}$ Amplatzer plug was deployed. Following embolization, flow into the hypoplastic portal vein improved with near complete occlusion of flow into the portosystemic shunts (Fig. 1c). The final portal venous gradient was $2 \mathrm{mmHg}$.

Enoxaparin was administered at a dose of $40 \mathrm{mg}$ daily for 90 days as prophylaxis against portal venous thrombosis. At 3 month follow up, the ammonia level was $133 \mathrm{mcmol} / \mathrm{L}$. Although still elevated, the patient's family and educators both subjectively noted mild improvements in her behavioral symptoms and described her as being more affectionate and less confrontational. A follow up CT demonstrated complete occlusion of the two portosystemic shunts, and a portal vein diminutive in caliber. The portal 
vein measured $7 \mathrm{~mm}$ in diameter on both pre and post-procedure CT scans. The total volume of the liver was found to be $843 \mathrm{~cm} 3$ on pre-procedure CT \& $1191 \mathrm{~cm} 3$ on post-procedure CT (Fig. 1d). The patient had no complications at 3 months of follow up.

\section{Conclusions}

This case demonstrated the feasibility and safety of treating two portosystemic shunts with a single staged endovascular closure with Amplatzer plugs. The increase in liver volume post-procedure is consistent with expected increase in trophic flow through the liver following closure of the two extrahepatic shunts. Previous reports describe both single and multi-staged closure, both by open surgery and by endovascular techniques. Previous experience suggests that a single stage approach may be safely performed if the portosystemic gradient remains $<10 \mathrm{~mm} \mathrm{Hg}$ to avoid complications of acute portal hypertension, and the hemodynamics measured in this patient allowed for closure of both shunts without staging.[3]

In conclusion, the management strategy of Type II Abernethy Syndrome should be to redirect blood flow into the hypoplastic native portal system, allowing for physiologic hepatic metabolism of splanchnic blood, hypertrophy of the portal system, and growth of the liver from the increased trophic flow.

\section{Abbreviations}

1. CT: computed tomography

2. IVC: inferior vena cava

3. F: French

4. DSA: digital subtraction angiography

\section{Declarations}

- Ethics approval and consent to participate: This case report was exempt of institutional board approval at the University of Kansas Medical Center.

- Consent for publication: Consent obtained from patient's family.

- Availability of data and materials: not applicable

- Competing interests: The authors have no financial or non-financial competing interests to disclose.

- Funding: These authors did not receive funding for this case report.

- Authors' contributions:

- Brenden Bombardier MD: Manuscript lead author

- Adam Alli MD: Manuscript co-author

- Aaron Rohr MD: Manuscript co-author 
- Zachary Collins MD: Attending interventional radiologist performing the case. Manuscript reviewer.

- Kavi Raval DO: Fellow interventional radiologist performing the case. Manuscript reviewer.

- Acknowledgements: none

\section{References}

1. Papamichail M, Pizanias M, Heaton N (2018) Congenital portosystemic venous shunt. Eur J Pediatr 177(3):285-294 i: 10.1007/s00431-017-3058-x[published Online First: Epub Date].. ( : do

2. Alonso-Gamarra E, Parrón M, Pérez A, Prieto C, Hierro L, López-Santamaría M (2011) Clinical and Radiologic Manifestations of Congenital Extrahepatic Portosystemic Shunts. A Comprehensive Review RadioGraphics 31(3):707-722 i: 10.1148/rg.313105070[published Online First: Epub Date]|. ; ): do

3. Rajeswaran S, Johnston A, Green J et al (2020) Abernethy Malformations: Evaluation and Management of Congenital Portosystemic Shunts. J Vasc Interv Radiol 31(5):788-794. doi:10.1016/j.jvir.2019.08.007[published Online First: Epub Date]|

\section{Figures}

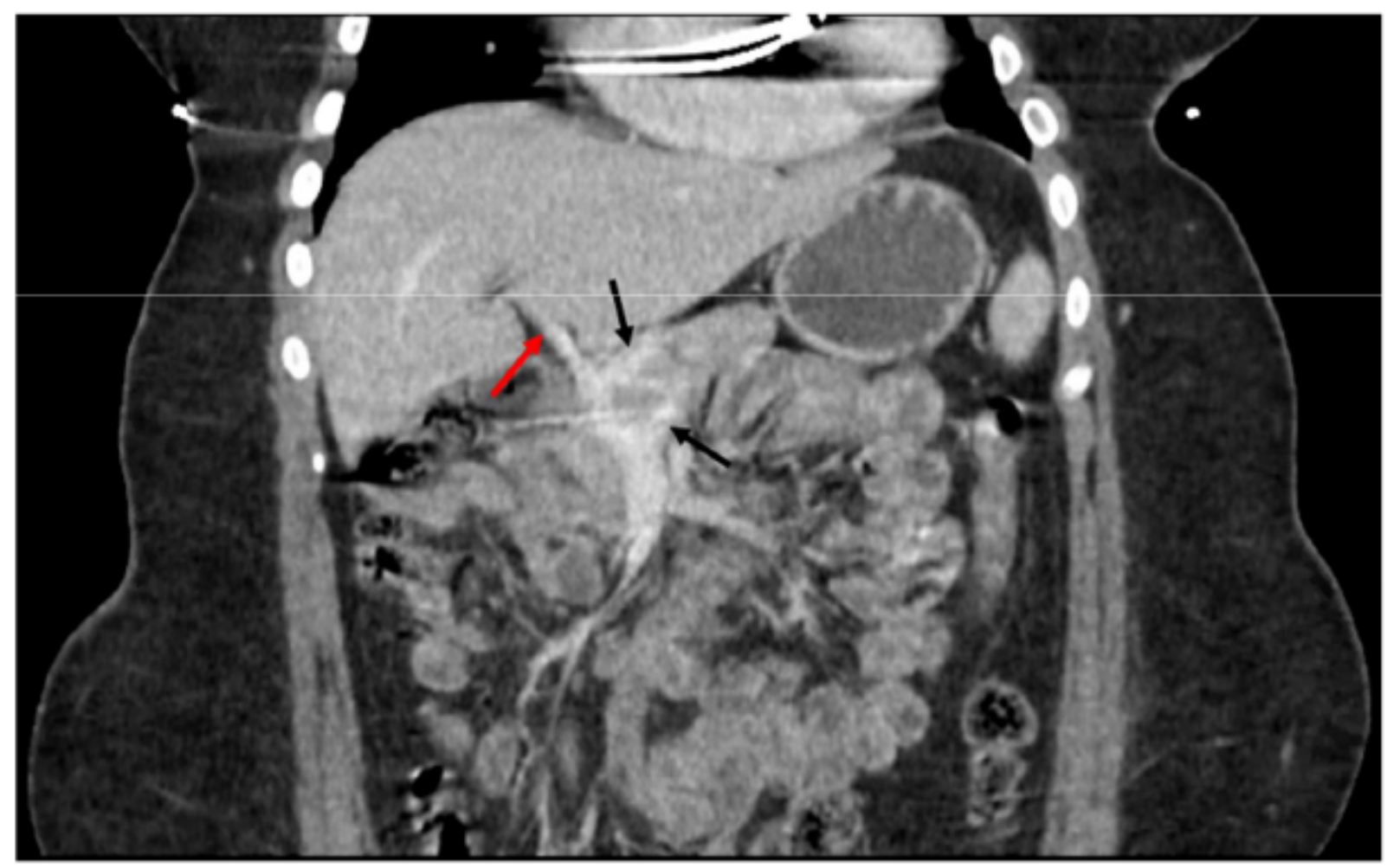

\section{Figure 1}


Coronal CT showing two extrahepatic portal shunts (black arrows) and a hypoplastic portal vein (red arrow).

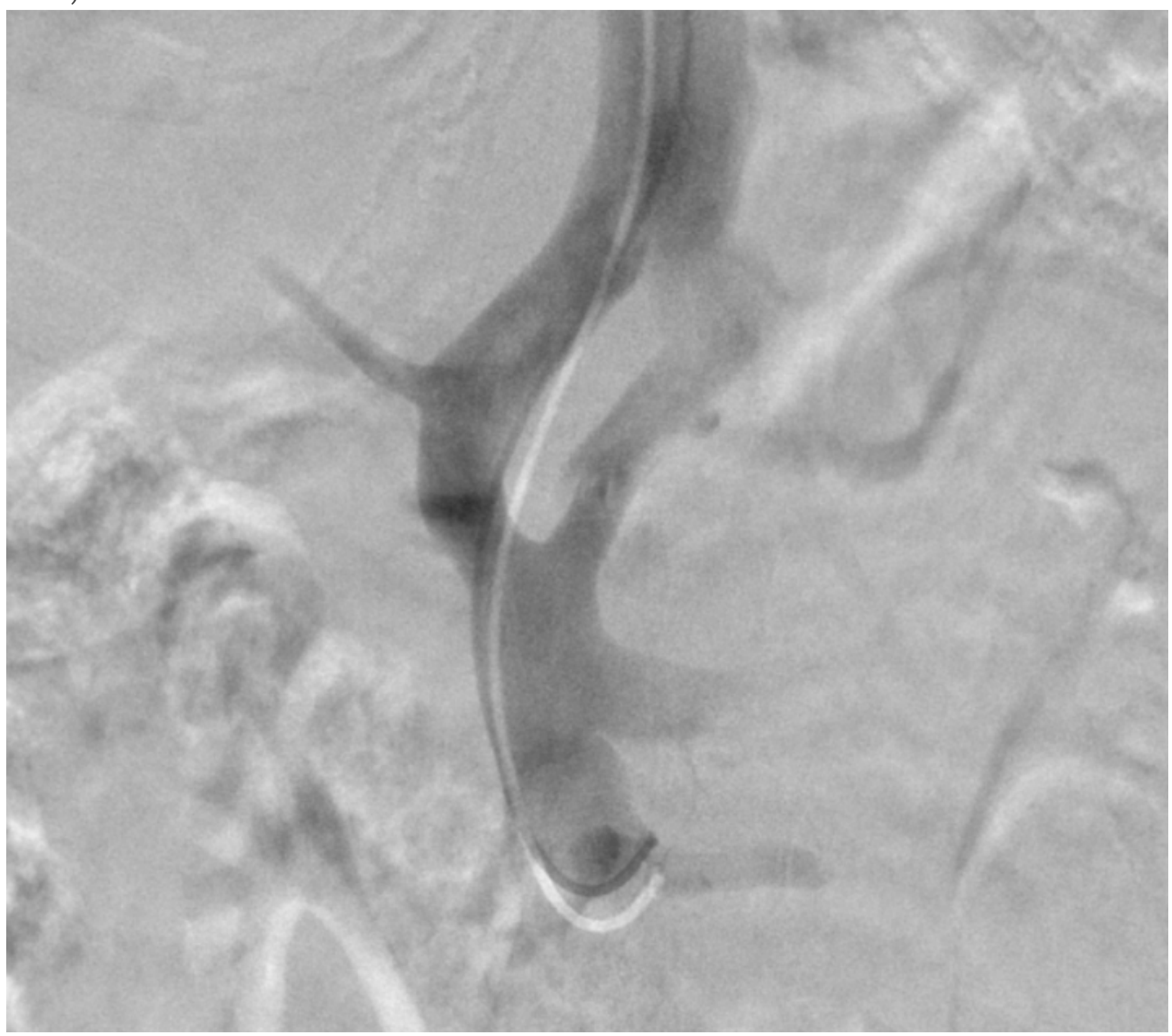

\section{Figure 2}

Splenic and superior mesenteric venography showed preferential flow through the shunts into the IVC without flow visualized into the intrahepatic portal vein. 


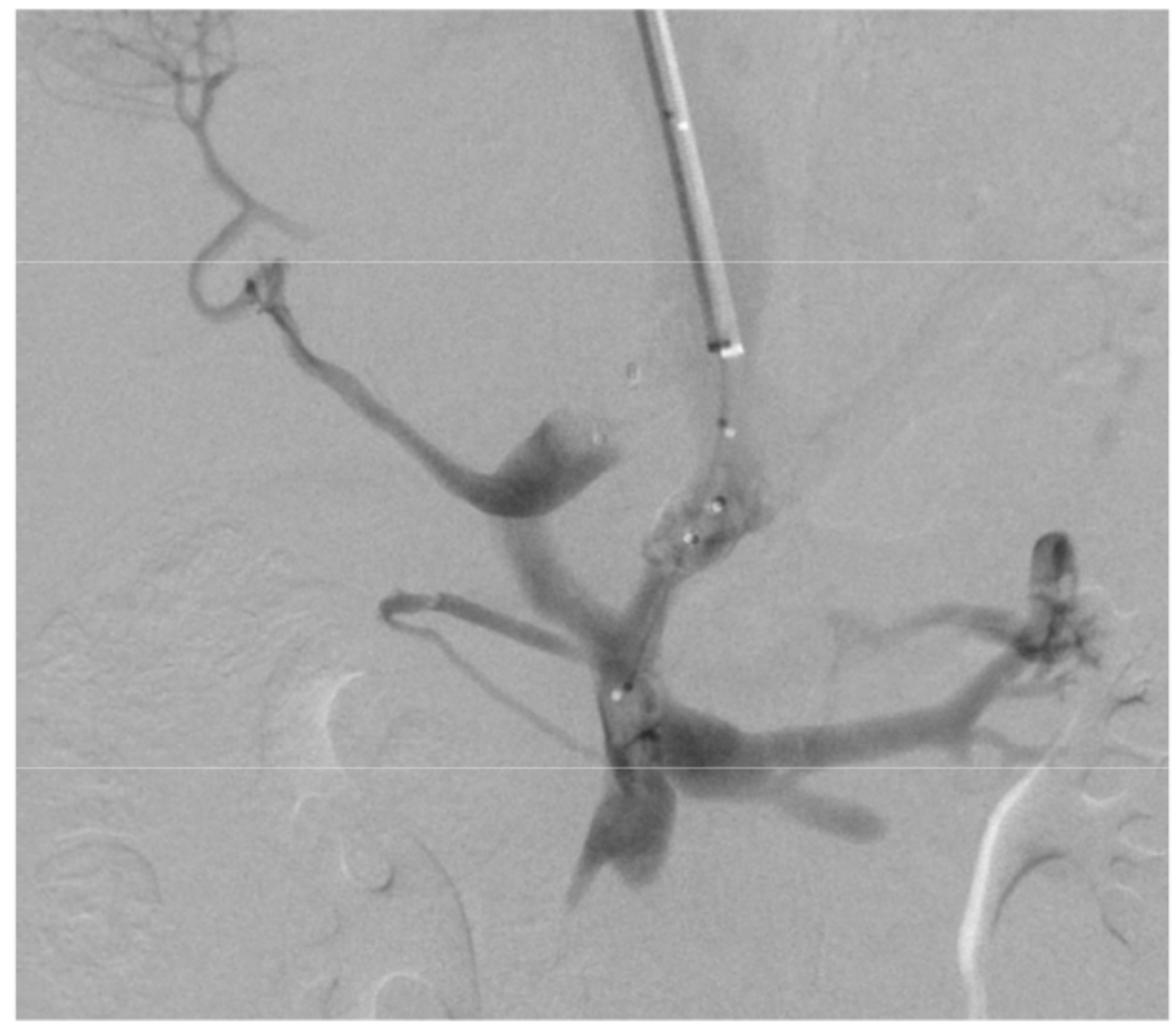

\section{Figure 3}

Portal venography following embolization showed increased flow into the intrahepatic portal vein with nearly complete occlusion of the portosystemic shunts. 